\title{
Intravascular Lymphoma Presenting as Myelopathy and Intracranial Hemorrhages
}

\author{
Kenta Orimo $^{1}$, Takuya Sasaki ${ }^{1}$, Yukio Kakuta ${ }^{2}$ and Ichiro Imafuku ${ }^{1}$
}

Key words: intravascular lymphoma (IVL), neuromyelitis optica (NMO), longitudinally extensive spinal cord lesions (LESCLs), susceptibility-weighted imaging (SWI), brain hemorrhages

(Intern Med 59: 3249, 2020)

(DOI: 10.2169/internalmedicine.5469-20)
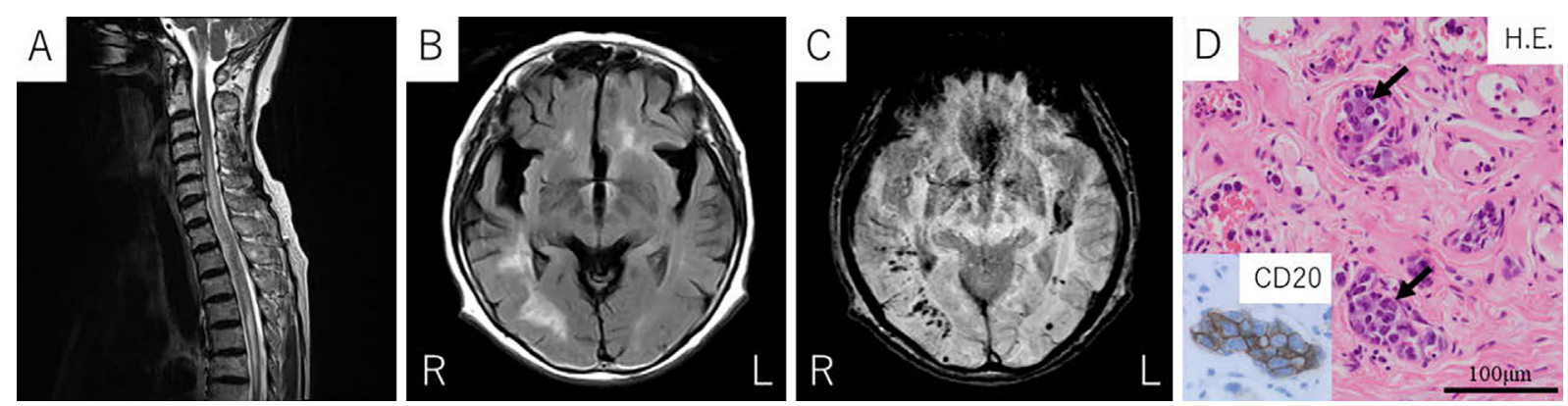

Picture.

An 82-year-old woman presented with subacute progression of paraplegia. Spinal magnetic resonance imaging (MRI) showed longitudinally extensive spinal cord lesions (LESCLs) on T2-weighted imaging (Picture A). Brain MRI showed multiple hyperintensity lesions on fluid-attenuated inversion recovery imaging (Picture B) with hemorrhagic lesions on susceptibility-weighted imaging (Picture $\mathrm{C}$ ). The patient was negative for the anti-aquaporin-4 antibody. A cerebrospinal fluid analysis showed no neoplastic cells, but her IL-10 level was elevated $(20 \mathrm{pg} / \mathrm{mL})$. A random skin biopsy revealed atypical lymphocytes (arrow) positive for CD 20 and CD79a in the subcutaneous vessels (Picture D). The patient was diagnosed with intravascular lymphoma (IVL) and given best supportive care. Myelopathy accounts for $5.78 \%$ of neurological complications of IVL (1). IVL can manifest as LESCLs, which can be a differential diagnosis of neuromyelitis optica (2). Intracranial hemorrhagic lesions are also reported in patients with IVL (3). One possible mechanism of hemorrhages is injury of the vessel walls caused by the infiltration of malignant cells into the small arterioles or venules (3). To our knowledge, our patient is the first case simultaneously exhibiting myelopathy and in- tracranial hemorrhages. A random skin biopsy should be considered when multiple intracranial hemorrhages exist in a patient with myelopathy.

The authors state that they have no Conflict of Interest (COI).

\section{References}

1. Fonkem E, Dayawansa S, Stroberg E, et al. Neurological presentations of intravascular lymphoma (IVL): meta-analysis of 654 patients. BMC Neurol 16: 9, 2016.

2. Kumar N, Keegan BM, Rodriguez FJ, Hammack JE, Kantarci OH. Intravascular lymphoma presenting as a longitudinally-extensive myelitis: diagnostic challenges and etiologic clues. J Neurol Sci 303: 146-149, 2011.

3. Passarin MG, Wen PY, Vattemi E, et al. Intravascular lymphomatosis and intracerebral haemorrhage. Neurol Sci 31: 793-797, 2010.

The Internal Medicine is an Open Access journal distributed under the Creative Commons Attribution-NonCommercial-NoDerivatives 4.0 International License. To view the details of this license, please visit (https://creativecommons.org/licenses/ by-nc-nd/4.0/).

\footnotetext{
${ }^{1}$ Department of Neurology, Yokohama Rosai Hospital, Japan and ${ }^{2}$ Department of Pathology, Yokohama Rosai Hospital, Japan Received: June 1, 2020; Accepted: June 22, 2020; Advance Publication by J-STAGE: August 4, 2020

Correspondence to Dr. Kenta Orimo, orimo-gun@umin.ac.jp
}

(C) 2020 The Japanese Society of Internal Medicine. Intern Med 59: 3249, 2020 